\title{
The spectral catalog of GRBs observed with INTEGRAL: IBIS/SPI joint spectral analysis
}

\author{
Ž. Bošnjak ${ }^{* a, b}$, D. Götz ${ }^{a}$, L. Bouchet ${ }^{c, d}$, S. Schanne ${ }^{a}$, B. Cordier $^{a}$ \\ ${ }^{a}$ AIM (UMR 7158 CEA/DSM-CNRS-Université Paris Diderot) Irfu/Service d'Astrophysique, \\ Saclay, F-91191 Gif-sur-Yvette Cedex, France \\ ${ }^{b}$ Department of Physics, University of Rijeka, 51000 Rijeka, Croatia \\ ${ }^{c}$ Université de Toulouse, UPS-OMP, IRAP, Toulouse, France \\ ${ }^{d}$ CNRS, IRAP, 9 Av. Colonel Roche, BP 44346, F-31028 Toulouse Cedex 4, France \\ E-mail: zeljka.bosnjak@cea.fr, gotz@cea.fr
}

We present the updated INTEGRAL catalog of gamma-ray bursts (GRBs) observed between November 2002 and February 2011. The catalog contains the spectral parameters for GRBs localized by the INTEGRAL Burst Alert System (IBAS). We used the data from the two main instruments on board the INTEGRAL satellite, the spectrometer SPI (SPectrometer on INTEGRAL), and the imager IBIS (the Imager on Board the INTEGRAL Satellite). The catalog contains the burst coordinates, positional errors, trigger times, durations and peak fluxes for 25 new GRBs obtained between September 2008 and February 2011. The spectral analysis was performed on the whole sample of GRBs observed by $I B I S$, including also the events occurring before September 2008. We applied a new data extraction technique developed in order to explore the energy regions of highest sensitivity for both instruments, $S P I$ and $I B I S$, and compared the prompt emission properties of INTEGRAL GRB sample with BATSE and Fermi samples.

"An INTEGRAL view of the high-energy sky (the first 10 years)" 9th INTEGRAL Workshop and celebration of the 10th anniversary of the launch,

October 15-19, 2012

Bibliotheque Nationale de France, Paris, France

${ }^{*}$ Speaker. 


\section{Introduction}

During the past two decades gamma-ray bursts (GRBs) have been observed by several missions providing the wealth of spectral and temporal data. The most complete catalogs of spectral GRB properties comprise the events observed by BATSE (Burst And Transient Source Experiment) onboard the Compton Gamma Ray Observatory [1], and the Swift satellite launched in 2004 [2]. The Fermi satellite launched in 2008 [3] has provided significant discoveries in the high energy (> $100 \mathrm{MeV}$ ) domain. In this paper we present the spectral catalog of the properties of gamma-ray bursts detected with INTEGRAL satellite [4]. We provide the general information of the previously unpublished events (25 GRBs observed after October 2008) in Table 1. In order to perform the spectral analysis, we combined the data from the two main instruments onboard INTEGRAL, the spectrometer $S P I$ and the imager IBIS.

\section{Method and Results}

The joint spectral analysis, using the data from both instruments, allows to perform the consistent analysis of the spectrum and exploits fully the potential of each instrument. The SPI data can provide the spectral information at energies where IBIS/ISGRI (INTEGRAL Soft Gamma-Ray Imager) effective area becomes low ( $200 \mathrm{keV}$ ), and therefore are suitable to determine the GRB spectral peak energy (typically at $\sim$ a few $100 \mathrm{keV}$ ). For the $S P I$ instrument, a spectrum for each of the 19 (where applicable) Ge detectors was computed. The net individual GRB spectra (i.e. onburst - off-burst spectra) have the advantage (with respect to the global spectra produced by OSA software) to be more accurate since the background spectra were computed for each GRB and each detector, taking into account the local temporal background evolution. For each SPI detector an individual response function was calculated, taking into account the GRB direction (either as determined by IBIS/ISGRI or by more precise X-ray or optical follow-up observations). This response function takes into account the exposed fraction of each detector for the GRB direction. For the IBIS/ISGRI spectra, due to the large number of detectors, we did not compute individual pixel spectra. We selected only the pixels that were fully illuminated by the GRB, in order to compute the off-burst and on-burst spectra. A corresponding spectral response was computed, taking into account the reduced $(\sim 30 \%)$ area of the detector plane we used. For each GRB we computed and fitted the time-integrated spectrum, using all the available SPI spectra and 1 ISGRI spectrum (Fig 1). We performed the spectral analysis of the time-integrated spectra for 63 GRBs on the energy interval $\sim 40 \mathrm{keV}-1 \mathrm{MeV}$. The spectral parameters of the prompt gamma-ray burst spectrum were compared with the results of the BATSE and Fermi missions. As an example, the distributions of the spectral peak energies and the low energy power law indices are shown in Figure 2. The majority of INTEGRAL GRBs have lower peak energies than the BATSE GRBs in the same fluence range, whereas there is a good agreement with the distribution of the Fermi sample. The distribution of the low energy power law slopes obtained for INTEGRAL GRBs is consistent with both, Fermi GBM and BATSE GRB samples.

\section{References}

[1] Fishman, G.J. et al. The first BATSE gamma-ray burst catalog, ApJSS 92 (1994) 229 
Table 1: Properties of INTEGRAL gamma-ray bursts detected between September 2008 and February 2011. Peak fluxes were measured in the energy band $20-200 \mathrm{keV}$.

\begin{tabular}{|c|c|c|c|c|c|c|c|c|c|}
\hline \multirow[t]{2}{*}{ GRB } & \multirow{2}{*}{$\begin{array}{c}\mathrm{t}_{\text {start }} \\
\text { (UTC) }\end{array}$} & \multirow{2}{*}{$\begin{array}{l}\text { R.A. } \\
\text { (deg) }\end{array}$} & \multirow{2}{*}{$\begin{array}{l}\text { Dec. } \\
\text { (deg) }\end{array}$} & \multirow{2}{*}{$\begin{array}{l}\text { Pos.error } \\
(\operatorname{arcmin})\end{array}$} & \multicolumn{2}{|c|}{ Afterglow } & \multirow[t]{2}{*}{$\mathrm{z}$} & \multirow{2}{*}{$\begin{array}{l}\mathrm{T}_{90} \\
(\mathrm{~s})\end{array}$} & \multirow{2}{*}{$\begin{array}{c}\text { Peak flux } \\
\left(\mathrm{ph} \mathrm{cm}^{-2} \mathrm{~s}^{-1}\right)\end{array}$} \\
\hline & & & & & X & $\mathrm{O}$ & & & \\
\hline 081003 & $13: 46: 01$ & 262.3750 & 16.5661 & 2.0 & Y & - & - & 25 & - \\
\hline 081003B & 20:48:08 & 285.0250 & 16.6914 & 2.0 & - & - & - & 24 & 3.13 \\
\hline 081016 & $06: 51: 32$ & 255.5708 & -23.3300 & 1.5 & $\mathrm{Y}$ & - & - & 32 & 7.34 \\
\hline 081204 & $16: 44: 56$ & 349.7750 & -60.2214 & 2.3 & $\mathrm{Y}$ & - & - & 13 & 0.75 \\
\hline 081226B & $12: 13: 11$ & 25.495 & -47.4389 & 2.5 & - & - & - & 0.55 & - \\
\hline 090107B & $16: 20: 38$ & 284.8075 & 59.5925 & 1.5 & $\mathrm{Y}$ & - & - & 20 & 1.47 \\
\hline 090625B & $13: 26: 21$ & 2.2625 & -65.7817 & 1.7 & $\mathrm{Y}$ & - & - & 10 & 1.93 \\
\hline 090702 & $10: 40: 35$ & 175.9 & 11.5 & 2.5 & Y & - & - & 19 & 0.06 \\
\hline 090704 & $05: 47: 50$ & 208.2042 & 22.79 & 2.5 & - & - & - & 76 & 1.31 \\
\hline 090814B & $01: 21: 14$ & 64.775 & 60.5828 & 2.9 & $\mathrm{Y}$ & - & - & 51 & 0.65 \\
\hline 090817 & $00: 51: 25$ & 63.9708 & 44.1244 & 2.6 & Y & - & - & 225 & 1.69 \\
\hline 091015 & $22: 58: 53$ & 306.1292 & -6.17 & 2.9 & - & - & - & 229 & 0.12 \\
\hline 091111 & $15: 21: 14$ & 137.8125 & -45.9092 & 2.9 & - & - & - & 354 & 0.03 \\
\hline 091202 & 23:10:08 & 138.8292 & 62.5439 & 2.5 & Y & $?$ & - & 40 & 0.08 \\
\hline 091230 & $06: 26: 53$ & 132.8875 & -53.8925 & 2.5 & Y & Y & - & 225 & 0.15 \\
\hline 100103A & $17: 42: 38$ & 112.3667 & -34.4825 & 1.6 & $\mathrm{Y}$ & - & - & 35 & 2.17 \\
\hline $100331 \mathrm{~A}$ & $00: 30: 23$ & 261.0625 & -58.9353 & 2.5 & - & - & - & 20 & 0.42 \\
\hline $100518 \mathrm{~A}$ & $11: 33: 38$ & 304.8000 & -24.5608 & 1.6 & Y & Y & 4 & 39 & 0.36 \\
\hline $100703 \mathrm{~A}$ & $17: 43: 37.37$ & 9.5208 & -25.7097 & 2.8 & - & - & - & 0.08 & - \\
\hline $100713 \mathrm{~A}$ & $14: 35: 52$ & 255.2083 & 28.3900 & 2.5 & $\mathrm{Y}$ & - & - & 25 & 0.16 \\
\hline 100909A & 09:04:06 & 73.9500 & 54.6544 & 2.0 & Y & Y & - & 66 & 0.26 \\
\hline 100915B & $05: 49: 36.4$ & 85.3958 & 25.0950 & 2.0 & - & - & - & 5.5 & - \\
\hline 101112A & $22: 10: 14$ & 292.2167 & 39.3589 & 1.3 & Y & Y & - & 24 & 7.60 \\
\hline 110112B & $22: 24: 54.7$ & 10.6000 & 64.4064 & 2.6 & - & - & - & 0.29 & - \\
\hline 110206A & 18:07:55 & 92.3417 & -58.8106 & 2.0 & Y & Y & - & 35 & - \\
\hline
\end{tabular}




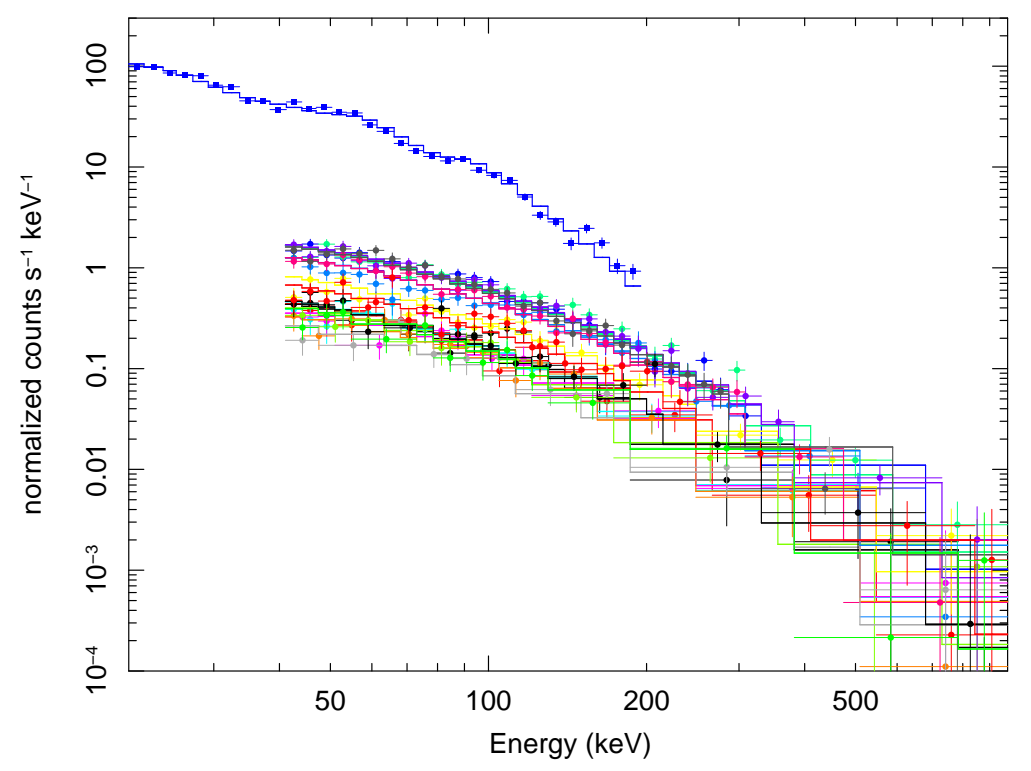

Figure 1: Spectral model and the data for GRB 061122. The dark blue squares are ISGRI data; the data on energy interval 20-200 keV were used for the fit. The coloured points are the data from 19 SPI detectors; the data covering the energies $40-1000 \mathrm{keV}$ were used in fitting procedure. The data were fitted with the cutoff-powerlaw model in this example.
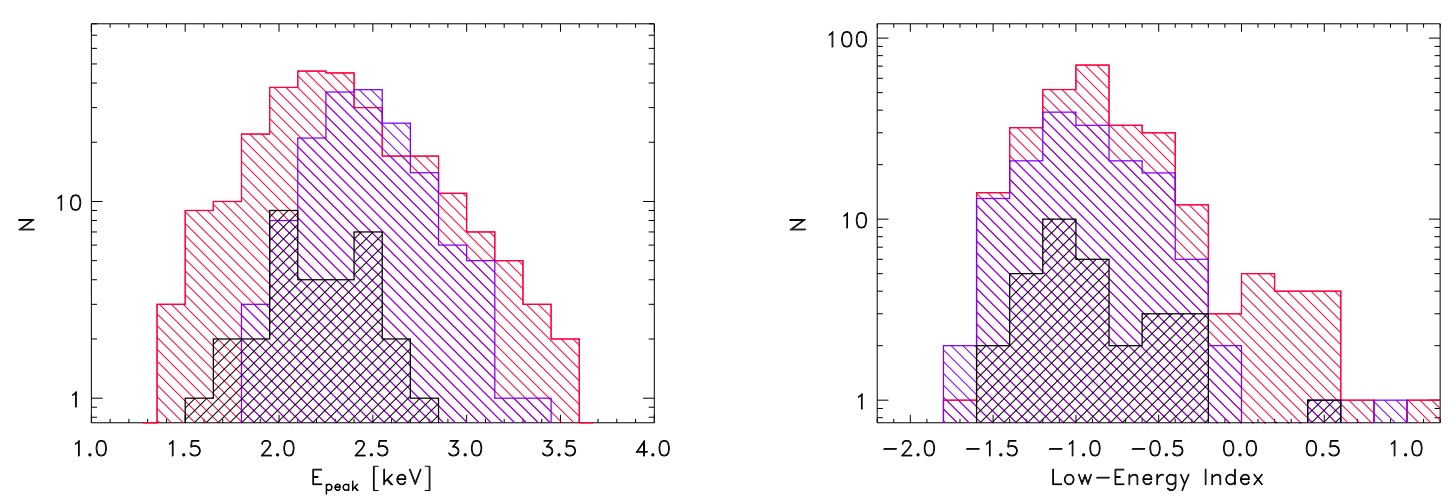

Figure 2: Distribution of the spectral peak energies and the low energy spectral powerlaw indices. The sample of INTEGRAL GRBs is shown in black; BATSE results (violet) and Fermi GBM results (red) of the time-integrated spectral analysis were used. For the comparison with the INTEGRAL sample of GRBs, only long events were selected fitted with the Band or cutoff powerlaw model, having the fluence in the same range as INTEGRAL GRBs.

[2] Gehrels, N. et al. The Swift Gamma-Ray Burst Mission, ApJ 611 (2004) 1005

[3] Atwood, W. B. et al. The Large Area Telescope on the Fermi Gamma-Ray Space Telescope Mission, ApJ 697 (2009) 1071

[4] Winkler et al. The INTEGRAL mission A\&A 411 (2003) L1 